\title{
The Černý conjecture and 1-contracting automata
}

\author{
Henk Don \\ Institute for Mathematics, Astrophysics, and Particle Physics \\ Faculty of Science, Radboud University Nijmegen \\ The Netherlands \\ henkdon@gmail.com \\ Submitted: Oct 6, 2015; Accepted: Jul 12, 2016; Published: Jul 22, 2016 \\ Mathematics Subject Classification: 68R05
}

\begin{abstract}
A deterministic finite automaton is synchronizing if there exists a word that sends all states of the automaton to the same state. Cerný conjectured in 1964 that a synchronizing automaton with $n$ states has a synchronizing word of length at most $(n-1)^{2}$. We introduce the notion of aperiodically 1-contracting automata and prove that in these automata all subsets of the state set are reachable, so that in particular they are synchronizing. Furthermore, we give a sufficient condition under which the Černý conjecture holds for aperiodically 1-contracting automata. As a special case, we prove some results for circular automata.
\end{abstract}

Keywords: Deterministic finite automaton; Synchronizing word.

\section{Introduction}

Let $\mathscr{A}=(Q, \Sigma, \delta)$ be a deterministic finite automaton (DFA), where $Q$ denotes the state set, $\Sigma$ the input alphabet, and $\delta: Q \times \Sigma \rightarrow Q$ the transition function. We denote the set of finite words over $\Sigma$ by $\Sigma^{\star}$. The transition function $\delta$ extends uniquely to a function $\delta: Q \times \Sigma^{\star} \rightarrow Q$.

The automaton $\mathscr{A}$ is called synchronizing if there exists a word $w \in \Sigma^{\star}$ and $q \in Q$ such that $\delta\left(q^{\prime}, w\right)=q$ for all $q^{\prime} \in Q$. The word $w$ is then said to be a synchronizing word for $\mathscr{A}$.

The following longstanding conjecture is due to Černý ([3], 1964):

Conjecture 1. If $\mathscr{A}$ is a synchronizing $n$-state automaton, then there exists a synchronizing word for $\mathscr{A}$ of length at most $(n-1)^{2}$.

Černý constructed for every $n$ an $n$-state automaton $\mathscr{C}_{n}$ in which the bound of his conjecture is attained. We show this automaton in the next example for the case $n=4$. 
Example 2. Consider the automaton $\mathscr{C}_{4}$ defined by the transition graph shown in Figure 1. The word $w=$ baaabaaab (having length 9) is synchronizing, since it maps all states to state 1 . Moreover, $w$ is the shortest synchronizing word for $\mathscr{C}_{4}$.

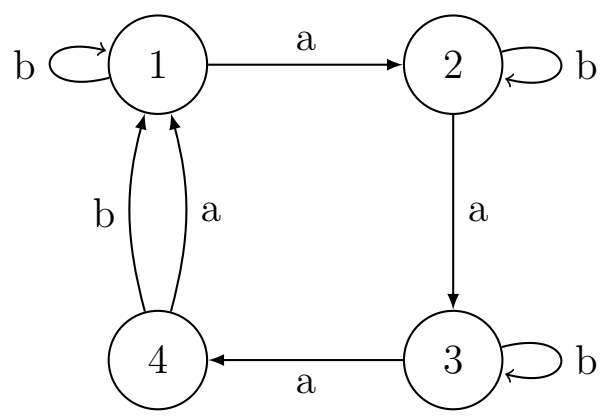

Figure 1: Transition graph of $\mathscr{C}_{4}$.

Černý himself provided an upper bound of $2^{n}-n-1$ for the length of the shortest synchronizing word. A substantial improvement was given by Starke [10], who was the first to give a polynomial upper bound, namely $1+\frac{1}{2} n(n-1)(n-2)$. The best known upper bound is still a cubic one, $\frac{1}{6}\left(n^{3}-n\right)$, that was established by Pin in 1983 [8]. He reduced proving this upper bound to a purely combinatorial problem which was then solved by Frankl [6]. Since then for more than 30 years no progress for the general case has been made.

The conjecture has been proved for some particular classes of automata, such as circular automata, aperiodic automata and one-cluster automata with prime length cycle. For these results and some more partial answers, see $[4,12,11,2,5,13,7,1]$. For a survey on synchronizing automata and the Černý conjecture, we refer to [14].

In this paper we look at $n$-state automata in which every $(n-1)$-subset of the state set $Q$ is reachable from $Q$. Such automata will be called 1-contracting. A word with the property that it maps $Q$ to an $(n-1)$-subset of $Q$ is called 1-deficient. If $w$ is a 1 -deficient word, the state that is not in the image of $w$ is said to be the excluded state. There also must be a unique state in the image which is reached twice by $w$. This state will be called the contracting state for $w$.

In a 1-contracting automaton, for every state $q$ there exists a 1-deficient word that excludes $q$. A collection $W$ of words is called 1-contracting if for all $q$ it contains exactly one word $w_{q}$ which excludes $q$. To such a collection we can associate a function $\sigma_{W}$ on $Q$ that maps each state $q$ to the unique contracting state for $w_{q}$. This function will be called the state map induced by $W$. If for some 1-contracting collection $W$ the state map is a cyclic permutation on $Q$, then the automaton is called aperiodically 1-contracting. For formal definitions and some more details, we refer to Section 2.

Our main results are presented in Section 3, where we will prove that aperiodically 1-contracting automata are synchronizing and that every nonempty subset of the state set is reachable. By imposing a (weak) restriction on the length of the 1-deficient words, we show that each $k$-subset is reachable by a word of length at most $n(n-k)$ which implies that the Cerný conjecture holds true in this case. 
In Section 4, we give some examples and discuss the scope of our results and their relation to circular automata. For circular automata it was shown by Dubuc ([4]) that if they are synchronizing, then the shortest synchronizing word has length at most $(n-$ $1)^{2}$. We give sufficient conditions for circular automata to be aperiodically 1-contracting. This means that for a subclass of circular automata, we prove synchronization. For this subclass, we also prove that the Cerný conjecture is satisfied and moreover that also here each $k$-subset is reachable by a word of length at most $n(n-k)$.

The class of aperiodically 1-contracting automata also contains automata satisfying a weaker notion of circularity: automata with a circle that uses multiple labels and goes through all states. We give an example that illustrates this and note that there is an infinite subfamily of such automata in which the Černý conjecture holds. Finally, we provide an example of an automaton that has no circle through all states, but still satisfies the Cerný conjecture because it falls into the framework of aperiodically 1-contracting automata, which demonstrates the extensiveness of this class. We finish our paper with a short discussion and a conjecture.

\section{1-contracting automata}

The transition function is naturally extended to a function on $2^{Q}$ by

$$
\delta(S, w)=\{\delta(q, w) \mid q \in S\}
$$

If $S \subseteq Q$ and $\delta(Q, w)=S$, we say that $S$ is reachable by $w$ (from $Q$ ). So an automaton $\mathscr{A}$ is synchronizing if and only if there exists a singleton which is reachable.

For $S \subseteq Q$, we define the inverse of the transition function under some word $w$ by

$$
\delta^{-1}(S, w)=\{q \mid \delta(q, w) \in S\} .
$$

For $w \in \Sigma^{\star}$ and $q \in Q$, we define the $w$-indegree of $q$ to be $\left|\delta^{-1}(q, w)\right|$. If $\left|\delta^{-1}(q, w)\right| \geqslant 2$, we say that $q$ is a contracting state for $w$.

In this paper, we study automata in which each $(n-1)$-subset of the state set is reachable from $Q$. Our main results concern aperiodically 1-contracting automata which are formally defined as follows.

Definition 3. Let $\mathscr{A}=(Q, \Sigma, \delta)$ be a DFA with $n$ states. $\mathscr{A}$ is called aperiodically 1contracting if there exist words $w_{1}, \ldots, w_{n} \in \Sigma^{*}$ and a cyclic order $q_{1} \prec q_{2} \prec \ldots \prec q_{n} \prec q_{1}$ on $Q$ such that for all $i=1, \ldots, n$ (and interpreting $q_{n+1}$ as $q_{1}$ )

$$
\delta\left(Q, w_{i}\right)=Q \backslash\left\{q_{i}\right\} \quad \text { and } \quad\left|\delta^{-1}\left(q_{i+1}, w_{i}\right)\right|=2 .
$$

In the remainder of this section we discuss the ideas behind this definition and we introduce some related terminology.

Definition 4. Let $\mathscr{A}=(Q, \Sigma, \delta)$ be a DFA. If a word $w_{q} \in \Sigma^{\star}$ satisfies

$$
\delta\left(Q, w_{q}\right)=Q \backslash\{q\},
$$

then $w_{q}$ will be called a 1-deficient word that excludes $q$. 
Definition 5. Let $\mathscr{A}=(Q, \Sigma, \delta)$ be a DFA with $n$ states. A collection $W \subseteq \Sigma^{\star}$ will be called a 1-contracting collection for $\mathscr{A}$ if for all $q \in Q$ it contains exactly one 1-deficient word that excludes $q$. If such a collection exists, $\mathscr{A}$ is called 1 -contracting. If $|w| \leqslant n$ for all $w \in W$, then $W$ is called an efficient 1-contracting collection.

Lemma 6. Every 1-contracting automaton $\mathscr{A}=(Q, \Sigma, \delta)$ admits an efficient 1-contracting collection.

Proof. Suppose $w_{q}$ is a 1-deficient word that excludes $q$. For every prefix $v$ of $w_{q}$, $|\delta(Q, v)| \geqslant\left|\delta\left(Q, w_{q}\right)\right|=n-1$. If $w_{q}$ has two different prefixes $u$ and $v$ such that $\delta(Q, u)=\delta(Q, v)$, then $w_{q}$ can be reduced by replacing the longer prefix by the shorter one. Since there are $n$ subsets of size $n-1, w_{q}$ can be reduced to have length at most $n$.

The previous lemma states that in a 1-contracting automaton on $n$ states, every $(n-1)$ set is reachable by a word of length at most $n$. In such automata, also many $(n-2)$-sets (in fact at least $\left(\begin{array}{l}n \\ 2\end{array}\right)-n$ ) are reachable by words of length at most $2 n$. The main goal is to show inductively that this behavior is inherited by sets of arbitrary size, i.e. that many $(n-k)$-sets are reachable by words of length at most $k n$. It turns out that it is not sufficient to merely have 1-contractivity, we also need some kind of aperiodicity, as the following example shows.

Example 7. Let $\mathscr{A}$ be the automaton with the transition graph given in Figure 2. The collection $W=\{c, c a, c a b, c b\}$ is an efficient 1-contracting collection for $\mathscr{A}$ since

$$
\begin{aligned}
\delta(Q, c) & =Q \backslash\{1\}, & & \delta(Q, c a b)=Q \backslash\{3\}, \\
\delta(Q, c a) & =Q \backslash\{2\}, & & \delta(Q, c b)=Q \backslash\{4\} .
\end{aligned}
$$

Thus, $\mathscr{A}$ is 1-contracting. However, $\mathscr{A}$ has no synchronizing word. For instance there does not exist a word $w \in \Sigma^{\star}$ such that $\delta(\{1,2\}, w)$ is a singleton.

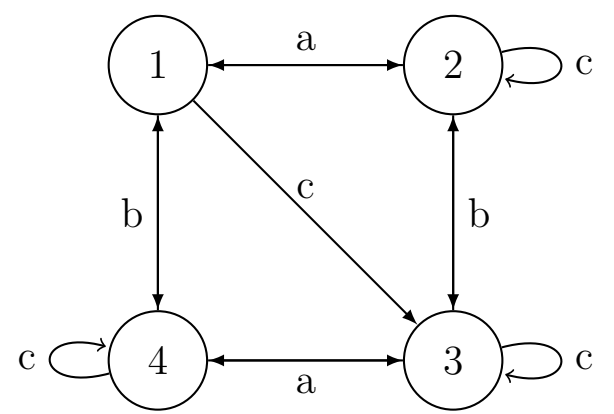

Figure 2: A 1-contracting automaton that fails to synchronize.

Our notion of aperiodicity will be formulated in terms of indegrees corresponding to the 1-deficient words. Suppose $\mathscr{A}$ is a 1-contracting automaton with $n$ states. Let $q \in Q$ and let $w_{q} \in \Sigma^{\star}$ be a 1-deficient word that excludes $q$. Then $\left|\delta^{-1}\left(q, w_{q}\right)\right|=0$ and $\left|\delta^{-1}\left(q^{\prime}, w_{q}\right)\right| \geqslant 1$ for all $q^{\prime} \neq q$. Since $\sum_{q \in Q}\left|\delta^{-1}\left(q, w_{q}\right)\right|=n$, there is a unique contracting state $q^{c}$ for $w_{q}$ which has $w_{q}$-indegree 2 . 
So in an automaton $\mathscr{A}$, a 1 -deficient word $w$ has a unique excluded state and a unique contracting state.

Definition 8. Let $\mathscr{A}$ be a DFA and let $W \subseteq \Sigma^{\star}$ be a 1-contracting collection for $\mathscr{A}$. Define the following collection of pairs of states $\Pi_{W} \subseteq Q \times Q$ :

$$
\Pi_{W}:=\left\{\left(q, q^{c}\right): \exists w \in W \text { s.t. }\left|\delta^{-1}(q, w)\right|=0,\left|\delta^{-1}\left(q^{c}, w\right)\right|=2\right\} .
$$

Let the map $\sigma_{W}$ from $Q$ to $Q$ be defined by

$$
\sigma_{W}(q)=q^{c} \Leftrightarrow\left(q, q^{c}\right) \in \Pi_{W}
$$

The map $\sigma_{W}$ will be called the state map induced by $W$.

Since for every $q \in Q$ there is exactly one $w \in W$ which excludes $q$, the state map induced by $W$ is well-defined.

Example 9. Consider the automaton $\mathscr{A}$ of Figure 2 with the 1-contracting collection $W=\{c, c a, c a b, c b\}$. The contracting states for the words in this collection follow directly from the equalities

$$
\left|\delta^{-1}(3, c)\right|=\left|\delta^{-1}(4, c a)\right|=\left|\delta^{-1}(1, c a b)\right|=\left|\delta^{-1}(2, c b)\right|=2 .
$$

So in this case $\Pi_{W}=\{(1,3),(2,4),(3,1),(4,2)\}$, and therefore the state map $\sigma_{W}: Q \rightarrow Q$ is defined by

$$
\sigma_{W}(1)=3, \quad \sigma_{W}(2)=4, \quad \sigma_{W}(3)=1, \quad \sigma_{W}(4)=2 .
$$

A DFA $\mathscr{A}$ is aperiodically 1-contracting if and only if there exists a 1-contracting collection $W \subseteq \Sigma^{\star}$ for which the induced state map $\sigma_{W}$ is a cyclic permutation on $Q$. As can be easily checked, the state map $\sigma_{W}$ of Example 9 is not a cyclic permutation.

\section{Synchronization of 1-contracting automata}

\subsection{Main results}

In this section we prove that in aperiodically 1-contracting automata all subsets of the state set are reachable by concatenating 1-deficient words. As a direct consequence, such automata are synchronizing.

Theorem 10. Let $\mathscr{A}=(Q, \Sigma, \delta)$ be an aperiodically 1-contracting DFA with $n$ states. Let $W \subseteq \Sigma^{\star}$ be a corresponding 1-contracting collection for which the state map $\sigma_{W}$ is a cyclic permutation on $Q$. Then every nonempty $k$-subset $S$ of $Q$ is reachable by a word $w_{S}$ of the form $w_{S}=w_{1} \ldots w_{n-k}, w_{i} \in W$. 
Corollary 11. Under the conditions of Theorem 10, $\mathscr{A}$ is synchronizing and there exist synchronizing words of the form $w=w_{1} \ldots w_{n-1}$.

Furthermore, we show that the Černý conjecture holds true if there is an efficient 1-contracting collection $W$ for which the state map is a cyclic permutation. By Lemma 6 , for $W$ to be efficient, it is sufficient if the 1-deficient words can be chosen to have minimal length.

Theorem 12. Let $\mathscr{A}=(Q, \Sigma, \delta)$ be an aperiodically 1-contracting DFA with $n$ states. If there exists an efficient 1-contracting collection $W \subseteq \Sigma^{\star}$ for which $\sigma_{W}$ is a cyclic permutation on $Q$, then

1. The shortest synchronizing word of $\mathscr{A}$ has length at most $(n-1)^{2}$.

2. For every nonempty set $S \subseteq Q$ of size $k$, there exists a word $w_{S}$ of length at most $n(n-k)$ such that $\delta\left(Q, w_{S}\right)=S$.

\subsection{Proofs of main results}

All main results are immediate consequences of the following key lemma.

Lemma 13. Suppose that an n-state automaton $\mathscr{A}=(Q, \Sigma, \delta)$ is aperiodically 1-contracting, i.e. it admits a 1-contracting collection $W$ such that the state map $\sigma_{W}$ is a cyclic permutation of $Q$. Then for each proper nonempty subset $S \subset Q$, there exists a word $w \in W$ such that $S=\delta(T, w)$ for some subset $T \subseteq Q$ with $|T|=|S|+1$.

Proof. For any fixed $q \in Q$, we have $Q=\left\{q, \sigma_{W}^{1}(q), \sigma_{W}^{2}(q), \ldots, \sigma_{W}^{n-1}(q)\right\}$. Since $S$ is a proper nonempty subset of $Q$, there exists $k \in\{0,1, \ldots, n-1\}$ such that $\sigma_{W}^{k}(q) \notin S$ while $\sigma_{W}^{k+1}(q) \in S$. Let $p=\sigma_{W}^{k}(q)$. By definition of the state map, $p$ is the excluded state for some word $w \in W$ and $\sigma_{W}(p)$ is the contracting state for this $w$. This means for $\sigma_{W}(p)=\sigma_{W}^{k+1}(q) \in S$ we have $\left|\delta^{-1}\left(\sigma_{W}(p), w\right)\right|=2$. Since the excluded state $p$ for $w$ does not lie in $S$, for each state $r \in S \backslash\left\{\sigma_{W}(p)\right\}$ we have $\left|\delta^{-1}(r, w)\right|=1$. Consequently, $\left|\delta^{-1}(S, w)\right|=|S|+1$ and we can take $T=\delta^{-1}(S, w)$.

Proof of Theorem 10. Let $S$ be an arbitrary $k$-subset of $Q, 1 \leqslant k<n$. By repeatedly applying Lemma 13, we get a sequence of words $w_{1}, w_{2}, \ldots, w_{n-k} \in W$ with the property that $\delta\left(Q, w_{1} w_{2} w_{n-k}\right)=S$.

Proof of Corollary 11. Theorem 10 directly implies that every singleton can be reached by a word of the form $w_{i_{1}} \ldots w_{i_{n-1}}$. So in fact there exist at least $n$ synchronizing words of this form.

Proof of Theorem 12. The second statement is a direct consequence of Theorem 10. Since the automaton is synchronizing, there exists at least one pair $\left\{q^{\prime}, q^{\prime \prime}\right\} \subseteq Q$ which can be mapped directly to a singleton. That is, for some label $a \in \Sigma$, we have $\left|\delta\left(\left\{q^{\prime}, q^{\prime \prime}\right\}, a\right)\right|=1$. The pair $\left\{q^{\prime}, q^{\prime \prime}\right\}$ is reachable by a word $w$ of length at most $n(n-2)$, so the word $w a$ is synchronizing and has length at most $n(n-2)+1=(n-1)^{2}$. 
Remark 14. The proof of Lemma 13 provides an algorithm to actually construct the synchronizing word $w a$ as defined in the proof of Theorem 12.

\section{1-contracting automata and circularity}

In this section we make some comments on the scope of our results and on the relation between 1-contractivity and circularity. An automaton is called circular if it has a label that cyclically permutes the states.

As a special case of 1-contracting automata we now consider circular automata having a label $a \in \Sigma$ of deficiency 1 , that is $|\delta(Q, a)|=|Q|-1$. Such automata are 1-contracting, since one can first read the 1-deficient label and then cyclically permute the excluded state. In this case, we have the following result:

Proposition 15. Let $\mathscr{A}=(Q, \Sigma, \delta)$ be a circular automaton with $n$ states. Suppose that $\mathscr{A}$ has a 1-deficient label $a$ and is circular with respect to $b$. Let $q_{a}$ be the state that is excluded by a and let $q_{a}^{c}$ be the unique contracting state for $a$. Denote the distance on the circle from $q_{a}$ to $q_{a}^{c}$ by $d$. If $\operatorname{gcd}(d, n)=1$, then

1. $\mathscr{A}$ is synchronizing.

2. The shortest synchronizing word of $\mathscr{A}$ has length at most $(n-1)^{2}$.

3. For every nonempty set $S \subseteq Q$ of size $k$, there exists a word $w_{S}$ of length at most $n(n-k)$ such that $\delta\left(Q, w_{S}\right)=S$.

Proof. Let $b$ be a label that cyclically permutes the states. Let $q_{1}=q_{a}$ and enumerate the other states according to their position on the circle. Let $W=\left\{w_{1}, \ldots, w_{n}\right\}$ be defined by $w_{i}=a b^{i-1}, 1 \leqslant i \leqslant n$. Then $W$ forms an efficient 1-contracting collection, since $\delta\left(Q, w_{i}\right)=Q \backslash\left\{q_{i}\right\}$. Note that the label $b$ rotates the excluded and contracting state simultaneously, preserving the distance. Therefore, after reading the word $w_{i}$, the distance on the circle between the excluded and contracting state is equal to $d$. This immediately determines the state map $\sigma_{W}$ :

$$
\sigma_{W}(q)=\delta\left(q, b^{d}\right), \quad \text { for all } q \in Q .
$$

Let $q \in Q$ and suppose $\operatorname{gcd}(d, n)=1$. Then $\sigma_{W}^{n}(q)=\delta\left(q, b^{n d}\right)=q$ and $\sigma_{W}^{k}(q)=\delta\left(q, b^{k d}\right) \neq$ $q$ if $1 \leqslant k<n$. In other words, $\sigma_{W}$ is a cyclic permutation on $Q$. Consequently the automaton is aperiodically 1-contracting and Theorem 12 applies.

Pin [9] already proved that if a circular automaton has a prime number of states and has a letter that is not a permutation, then it is synchronizing and has a synchronizing word of length at most $(n-1)^{2}$. Our proposition is slightly different from Pin's result: we need stronger assumptions on the letter that is not a permutation, but we allow a composite number of states. Dubuc's result (the maximal length $(n-1)^{2}$ holds for every synchronizing circular automaton, [4]) also has weaker premises on the letters, but does 
assume synchronization. Compared to these two results, we get the additional property that all $k$-sets are reachable with words of length at most $n(n-k)$.

In contrast with previous results on automata with some circular structure, the class of 1-contracting automata allows for circles defined by a word consisting of different letters. To illustrate this, we give an example.

Example 16. Consider the automaton $\mathscr{A}$ of Figure 3, where for simplicity we did not include the selfloops. In this automaton, the labels $a$ and $b$ are both permutations, and together they make a circle through all states. With only these two labels the automaton would not be synchronizing. Therefore there is also a label $c$ which is 1-deficient. Let $W$ be the collection of the words in the tables below. These words are 1-deficient and their excluded and unique contracting states are indicated.

\begin{tabular}{c|c|c} 
word & excl. & contr. \\
\hline$w_{1}=c$ & 1 & 2 \\
$w_{2}=c a$ & 2 & 3 \\
$w_{3}=c a b$ & 3 & 4
\end{tabular}

\begin{tabular}{c|c|c} 
word & excl. & contr. \\
\hline$w_{4}=c a b^{2}$ & 4 & 5 \\
$w_{5}=c a b^{3}$ & 5 & 6 \\
$w_{6}=c a b^{3} a$ & 6 & 1
\end{tabular}

$W$ is an efficient 1-contracting collection. Clearly the state map $\sigma_{W}$ is a cyclic permutation, so it follows that $\mathscr{A}$ is aperiodically 1-contracting. By Theorem 12, all k-subsets of the state set are reachable by words of length at most $6(6-k)$. In particular, this automaton satisfies the Černy conjecture.

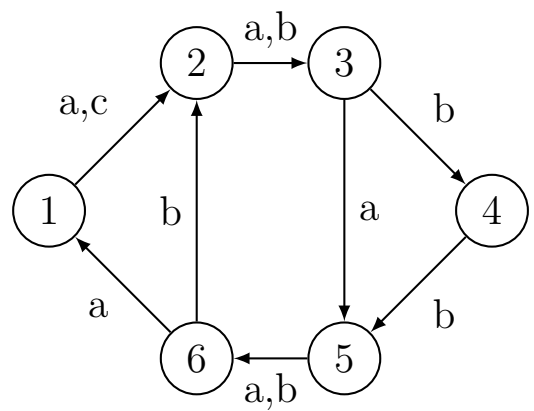

Figure 3: An automaton where the labels $a$ and $b$ together make a circle.

Observe that the previous example can be easily extended to an infinite family of aperiodically 1-contracting automata that have a circle with multiple labels. Theorem 12 applies as long as the excluded state and the contracting state move simultaneously along the circle on a mutual distance that is relative prime to the number of states, while the other states are permuting.

As a final illustration, we give an example that shows that our results are not restricted to automata with a cycle through all states. 
Example 17. The automaton in Figure 4 is aperiodically 1-contracting with the following collection $W$ of 1-deficient words:

\begin{tabular}{c|c|cc|c|c} 
word & excl. & contr. & word & excl. & contr. \\
\hline$w_{1}=a$ & 1 & 2 & $w_{3}=a b c$ & 3 & 1 \\
$w_{2}=a b$ & 2 & 4 & $w_{4}=a c$ & 4 & 3
\end{tabular}

Here the state map $\sigma_{W}$ is given by $\sigma_{W}(1)=2, \sigma_{W}(2)=4, \sigma_{W}(3)=1, \sigma_{W}(4)=3$, which defines a cyclic permutation on $Q$. So also here Theorem 12 applies: all $k$-subsets of the state set are reachable with a word of length at most $4(4-k)$ and the Črny conjecture holds true. In fact the word acabca is a synchronizing word that is obtained by concatenating three of the 1-deficient words $w_{i}$.

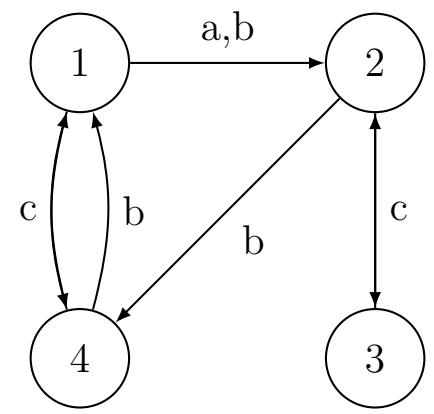

Figure 4: An aperiodically 1-contracting automaton without a full circle.

\section{Conclusion and perspectives}

We have introduced the notion of 1-contracting automata in terms of the existence of 1-deficient words. We formulated an aperiodicity property and have shown that this property suffices to prove synchronization. If the 1-deficient words with this property have length at most $n$, then the automaton satisfies the Cerný conjecture. In particular this is the case if the state map induced by a collection of minimal 1-deficient words is a cyclic permutation.

We believe that some elements in our definitions are not really essential for the results, and that they only help in organizing a clear proof. So a natural question is if the conditions can be weakened, while still exploiting the ideas we presented in this paper. For instance, one would expect that under a suitable aperiodicity condition a synchronizing word of length at most $(n-1)^{2}$ exists for automata in which every $(n-1)$-set has a subset which is reachable by a word of length at most $n$.

We proved for the class of aperiodically 1-contracting automata that each reachable subset of size $k$ is reachable by a word of length at most $n(n-k)$ if there exists an efficient 1-contracting collection. In fact we believe that this is a universal property, so we finish our paper with this conjecture:

Conjecture 18. Let $\mathscr{A}=(Q, \Sigma, \delta)$ be an $n$-state automaton. If $S \subseteq Q$ is a set of size $k$ and there exists a word $w$ such that $\delta(Q, w)=S$, then there exists a word with this property of length at most $n(n-k)$. 
This conjecture would imply the Černý conjecture, since in a synchronizing automaton there always exists a pair of states that can be mapped to a singleton by some letter $a \in \Sigma$.

\section{Acknowledgement}

The author is grateful to an anonymous referee for his comments, especially for suggesting Lemma 13 which significantly shortened and simplified the proofs of the main results.

\section{References}

[1] J. Almeida, S. Margolis, B. Steinberg, M. Volkov. Representation theory of finite semigroups, semigroup radicals and formal language theory. Transactions of the American Mathematical Society, 361, 1429-1461, 2009.

[2] M.-P. Béal, M.V. Berlinkov, D. Perrin. A quadratic upper bound on the size of a synchronizing word in one-cluster automata. Int. J. Foundations Comput. Sci. 22, 277-288, 2011.

[3] J. Černý. Poznámka k homogénnym experimentom s konečnými automatmi. Matematicko-fyzikálny časopis, Slovensk. Akad. Vied, Vol. 14, No. 3, 208-216, 1964.

[4] L. Dubuc. Sur les automates circulaires et la conjecture de Černý. RAIRO Inform. Theor. Appl. 32, 21-34, 1998.

[5] D. Eppstein. Reset sequences for monotonic automata. SIAM Journal on Computing, 19, 500-510, 1990.

[6] P. Frankl. An extremal problem for two families of sets. European Journal of Combinatorics, 3, 125-127, 1982.

[7] J. Kari. Synchronizing finite automata on Eulerian digraphs. Theoretical Computer Science, 295 (1-3), 223-232, 2003.

[8] J.-E. Pin. On two combinatorial problems arising from automata theory. Annals of Discrete Mathematics, 17, 535-548, 1983.

[9] J.-E. Pin. Sur un cas particulier de la conjecture de Černý. Automata, languages and programming (Fifth Internat. Colloq., Udine, 1978), Lecture Notes in Comput. Sci., 62, Springer, Berlin-New York, 345-352, 1978.

[10] P.H. Starke. Eine Bemerkung über homogene Experimente. Elektronische Informationverarbeitung und Kybernetik, 2, 257-259, 1966.

[11] B. Steinberg. The Černý conjecture for one-cluster automata with prime length cycle. Theoretical Computer Science, 412 (39), 5487-5491, 2011.

[12] A. Trahtman. The Cerný conjecture for aperiodic automata. Discrete Mathematics and Theoretical Computer Science, Vol. 9, No.2, 3-10, 2007.

[13] M.V. Volkov. Synchronizing automata preserving a chain of partial orders. Theoretical Computer Science, 410 (37), 3513-3519, 2009.

[14] M.V. Volkov. Synchronizing automata and the Černý conjecture. LATA (2008) $11-$ 27, Lecture Notes in Comput. Sci., 5196, Springer, Berlin, 2008. 